\title{
MINOR COMPONENT EXTRACTION FROM PALM METHYL ESTER USING POTASSIUM CARBONATE GLYCEROL BASED DEEP EUTECTIC SOLVENT (DES)
}

\author{
R. Manurung ${ }^{1, *}$ and Alexander ${ }^{1}$ \\ ${ }^{1}$ Department of Chemical Engineering, University of Sumatera Utara, \\ Padang Bulan-20222, Medan, Indonesia \\ *Email: renita.manurung@usu.ac.id
}

\begin{abstract}
Biodiesel is well known as an alternative to petroleum fuel diesel and has an advantage over petroleum diesel such as lower exhaust emission and renewability, however, the high cost of biodiesel production still becomes an obstacle to overcome. The extraction of valuable minor components from methyl esters, such as vitamin E and carotenoids, was done to reduce the production cost of biodiesel. Biodiesel (methyl ester) was made by using a transesterification reaction, which involves the reaction of Crude Palm Oil (CPO) and methanol. Methyl ester was then separated from the by-product, glycerol. The methyl ester was then mixed with potassium carbonate-glycerol based Deep Eutectic Solvent (DES) to extract the minor components. DES usage to extract minor components showed that DES can be used to extract vitamin $\mathrm{E}$ and carotenoids; while purifies the methyl ester simultaneously.
\end{abstract}

Keywords: Biodiesel, Deep Eutectic Solvent, Extraction, Carotenoids, Vitamin E

๑ RASĀYAN. All rights reserved

\section{INTRODUCTION}

Biodiesel is a renewable fuel substitute for diesel fuel that is mostly made from animal and vegetable fats, which can be mixed or used directly in diesel engines due to its similar characteristics with diesel fuel ${ }^{1}$.Transesterification is the most common way to produce biodiesel by reacting triglycerides of vegetable oils and a short-chain alcohol (e.g. methanol or ethanol) $)^{2,3}$. Glycerol is the by-product of biodiesel synthesis ${ }^{4}$.Methanol is the most used alcohol in transesterification reaction due to its high reactivity, low energy requirement and fast reaction time ${ }^{5}$. Biodiesel has an advantage over petroleum diesel, such as renewability, lower gas emission (burn up to $70 \%$ cleaner), better fuel properties and biodegradable, but on the other side, the high cost of biodiesel production makes it less competitive compared to petroleum diesel ${ }^{2,6}$.

Crude Palm Oil (CPO) is oil extracted from the mesocarp of the fruit of the oil palm tree. Indonesia, as the leading producer of $\mathrm{CPO}$, has an abundant stock of $\mathrm{CPO}$, therefore $\mathrm{CPO}$ is chosen as the raw material for methyl ester (biodiesel) production. CPO consists more than $90 \%$ wt. of triglyceride ${ }^{7,8}$. Besides that, CPO also contains valuable minor components, such as vitamin E, carotenoids and phytosterols, that are not involved in transesterification reaction ${ }^{8,9}$. These minor components contribute to the antioxidant activity of the palm oil ${ }^{10}$.Therefore, the extraction of these valuable minor components was done in order to lower the production cost of biodiesel.

One of the extraction methods is by using a Deep Eutectic Solvent (DES) to extract the minor components from raw methyl ester. DESs is an advanced generation of Ionic Liquid (IL) which are biodegradable, cheap and easy to prepare, while still having IL properties, such as low melting point, low volatility, high thermal stability, high polarity, the inflammable and wide range of solubility ${ }^{11}$. DESs are formed by mixing organic halide salts with an organic compound. Organic halide salts act as Hydrogen Bond Acceptor (HBA) while the organic compound act as Hydrogen Bond Donor (HBD), hence hydrogen bond is formed between those compounds ${ }^{12}$.

Rasayan J. Chem., 11(4), 1519-1524(2018)

http://dx.doi.org/10.31788/RJC.2018.1143079

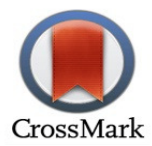


A recent study done by Hadi et al (2015) had demonstrated the use of DES to extract minor components, specifically vitamin E, from CPO. Hadi Et al (2015) used choline chloride-based DES with acetic acid, malonic acid, and citric acid as the Hydrogen Bond Donor (HBD) ${ }^{13}$. However, the use of DES to extract minor components from methyl ester from CPO is yet reported.

In this work, DES was produced from potassium carbonate and glycerol and was used to extract the minor components, such as vitamin $\mathrm{E}$ and carotenoids, from raw methyl ester produced from transesterification reaction.

\section{Materials}

\section{EXPERIMENTAL}

Crude Palm Oil was obtained from PTPN IV, methanol $\left(\mathrm{CH}_{3} \mathrm{OH}\right)$, potassium hydroxide $(\mathrm{KOH})$, potassium carbonate $\left(\mathrm{K}_{2} \mathrm{CO}_{3}\right)$ and glycerol $\left(\mathrm{C}_{3} \mathrm{H}_{8} \mathrm{O}_{3}\right)$ were obtained from commercial sources and used in the synthesis of methyl ester and DES.

\section{DES Synthesis}

Potassium carbonate and glycerol with a molar ratio of 1:5; 1:6 and 1:7 were mixed, and the mixtures were stirred at $80^{\circ} \mathrm{C}$ at a stirring speed of $300 \mathrm{rpm}$ for 1 hour until homogenous and transparent liquids are formed.

\section{Transesterification of Palm Oil}

Potassium hydroxide (1\% wt. of CPO) was suspended in methanol and shaken until it formed homogenous potassium methoxide. Afterward, potassium methoxide was added to CPO with 1:9 molar ratio of CPO to methanol in a batch reactor with $400 \mathrm{rpm}$ mixing speed at $65^{\circ} \mathrm{C}$ for 80 minutes. The products were transferred to a separation funnel and after 15 minutes settling, raw methyl ester phase (upper layer) was separated from the glycerol-rich phase (lower phase). Methyl ester phase was washed using a wet washing method.

\section{Extraction of Minor Components}

Ten grams of methyl ester obtained from transesterification reaction were mixed with $100 \mathrm{ml}$ of $\mathrm{n}$-hexane, while $100 \mathrm{ml}$ methanol was mixed with a certain amount of DES to lower its viscosity. Both of these mixtures then were mixed in a batch reactor with $400 \mathrm{rpm}$ mixing speed for three hours. The products were transferred to a separation funnel and after two hours settling, the upper layer was separated from the lower phase, which contained valuable minor components. The lower phase was washed using a mixture of watern-hexane mixtures with a volume ratio of $4: 1$ and was settled until two layers were obtained. The upper layer, which contained minor components and $\mathrm{n}$-hexane, was evaporated in a rotary evaporator to separate n-hexane from the extract.

\section{Product Analysis}

The content of vitamin E in the extract was analyzed using High-Performance Liquid Chromatography (HPLC). The content of carotenoids remained in the methyl ester was analyzed using Spectrophotometer UV-Vis. The purity of methyl ester was analyzed using Gas Chromatography (GC).

\section{RESULTS AND DISCUSSION \\ Effect of DES on Vitamin E Concentration Extracted from Palm Methyl Ester}

Extraction of vitamin E from palm methyl ester was done by using DES. DES can be used to extract the vitamin $E$ due to the presence of hydroxyl group in vitamin E molecules. DES has a high affinity to bind with a hydroxyl group. Hydrogen bond then is formed between the hydroxyl group and ammonium salt from DES. Therefore, vitamin E can be extracted from methyl ester using DES. ${ }^{14}$

As indicated by Fig.-2, the highest concentration of vitamin $\mathrm{E}$ was achieved by using the highest molar ratio of potassium carbonate to glycerol (1:7) in DES and the highest mass ratio of ester to DES (1:4) for the extraction process, which $406,10 \mathrm{ppm}$ of vitamin $\mathrm{E}$ was obtained.The addition of glycerol in DES synthesis decreases the viscosity and density of the DES, therefore making it a more suitable solvent.The addition of DES will increase the number of DES molecules that form a hydrogen bond with the hydroxyl group of vitamin $\mathrm{E}$, hence increasing its extraction effectivity ${ }^{14}$. 
From this result, it can be concluded that DES can extract vitamin E from methyl ester by forming a hydrogen bond with vitamin E.

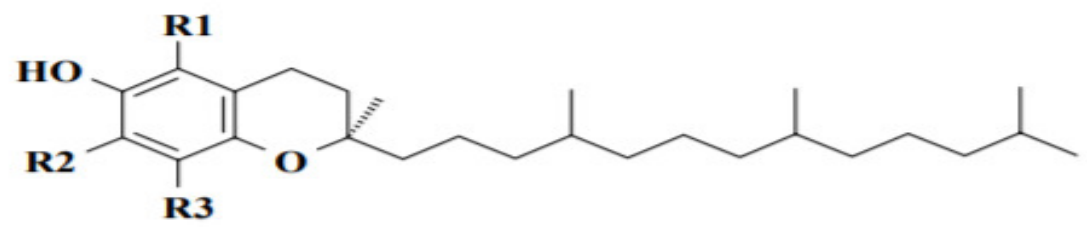

Tocopherol

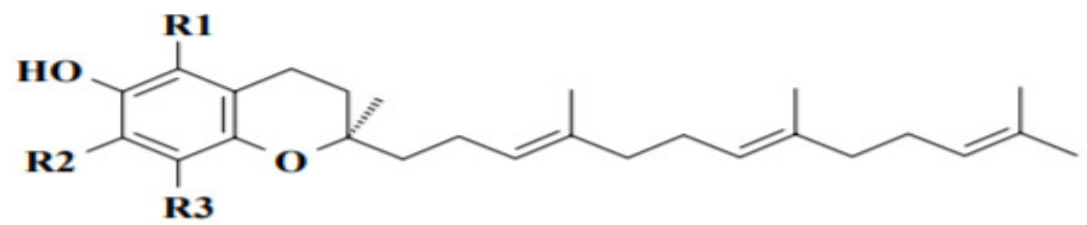

Tocotrienol

Fig.-1: Hydroxyl Group in Vitamin E Molecules ${ }^{15}$

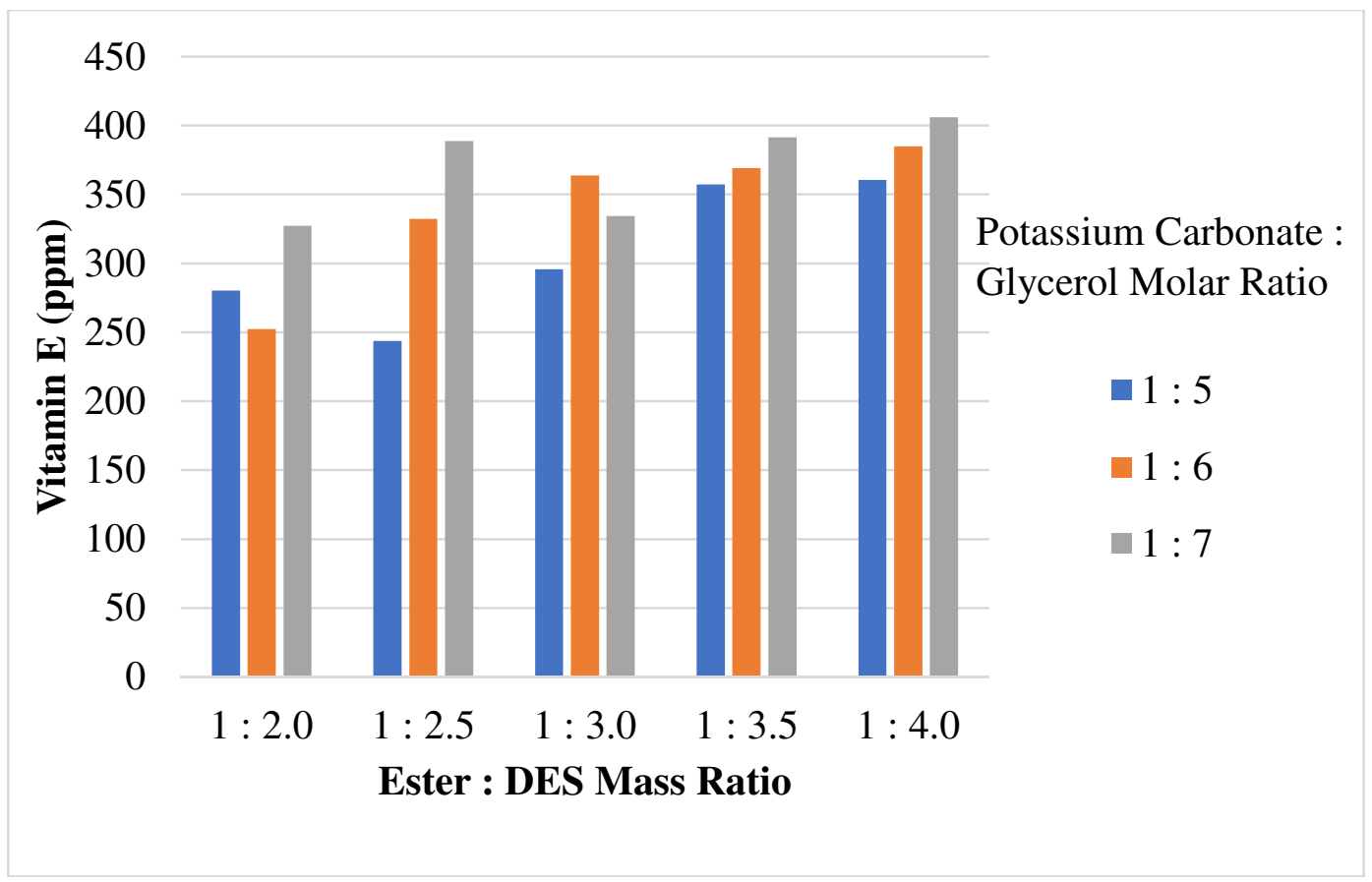

Fig.-2: The Effect of DES on Vitamin E Concentration

\section{Effect of DES on Carotenoids Concentration Remained in Methyl Ester}

Carotenoids in CPO are divided into two main groups, carotenes, and xanthophylls. Carotenes are carotenoids without hydroxyl group in their molecules, while xanthophylls have a hydroxyl group in their molecules. Carotenoids in CPO consist mostly of carotenes, hence carotenoids extraction from palm methyl ester is ineffective. This is due to DES inability to form a hydrogen bond with carotenes, however, DES is able to extract xanthophylls from palm methyl ester because of the presence of hydroxyl group in their molecules ${ }^{16,17}$.

As indicated by Fig.-4, analysis results of carotenoids concentration remained in methyl ester are fluctuating, this is due to insignificance amount of xanthophylls in CPO. In this study, the lowest 
RASĀYAN J. Chem.

Vol. 11 | No. 4 |1519 - 1524| October - December | 2018

concentration of carotenoids remained in methyl ester was achieved using the 1:5 molar ratio of potassium carbonate to glycerol and the 1:4 mass ratio of ester to DES, which result was $376.0 \mathrm{ppm}$ of carotenoids remained in methyl ester.

From this result, it can be concluded that DES is not effective in extracting carotenoids from palm methyl ester, due to the absence of hydroxyl group in carotenes, while most of CPO carotenoids consist of carotenes.
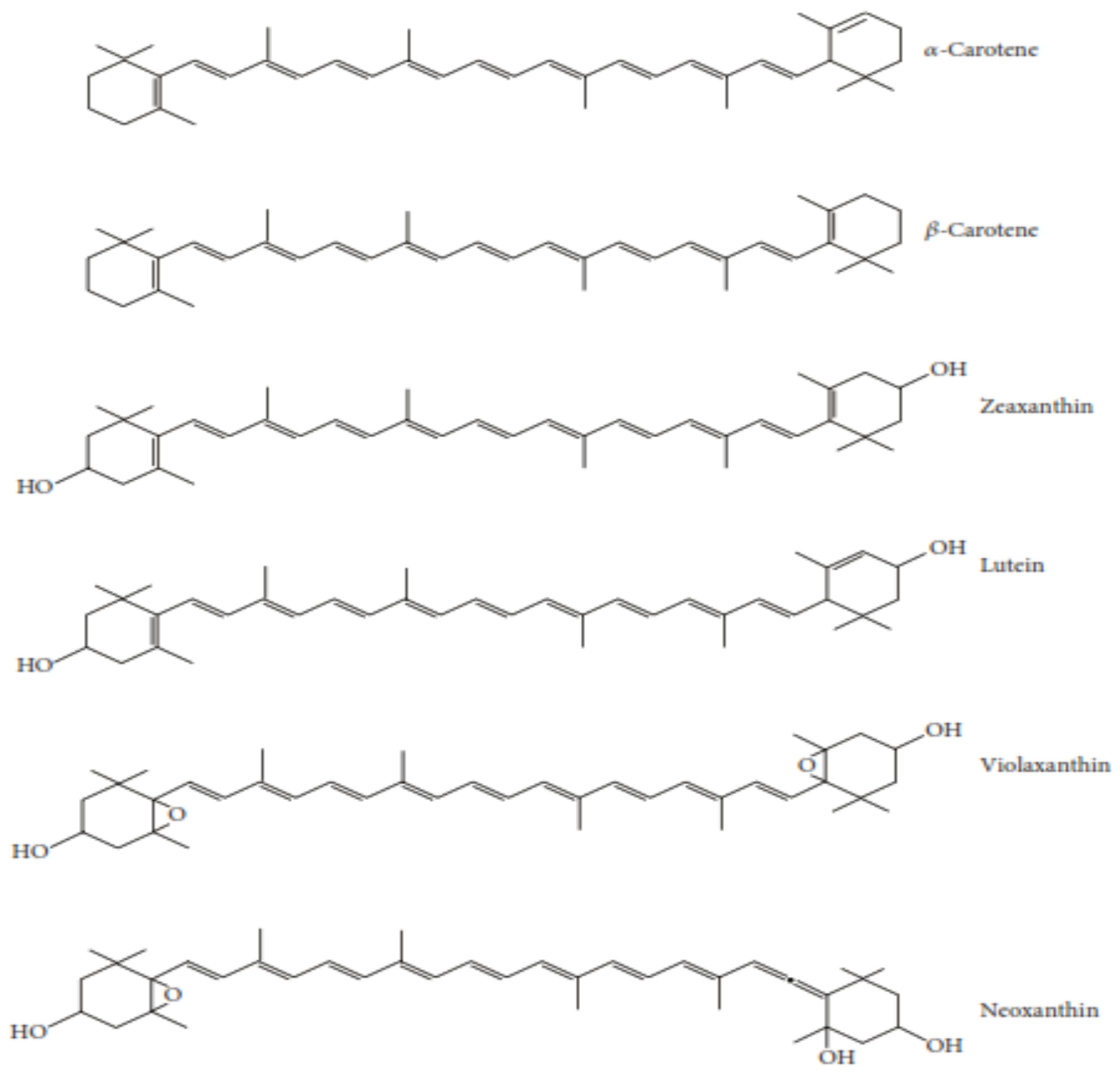

Fig.-3: Carotenoids Molecular Structure ${ }^{17}$

\section{Effects of DES on Methyl Ester Purity}

Aside from its ability to extract valuable minor components from methyl ester, such as vitamin $\mathrm{E}$ and carotenoids, DES also can be used to extract impurities components, such as unreacted alcohol, catalyst, glycerol, free fatty acid, monoglycerides and diglycerides. This is due to the presence of hydroxyl group in their molecules, hence DES will form a hydrogen bond with their hydroxyl group ${ }^{14}$.

Fig. -5 shows that extraction using DES increases the overall methyl ester purity from $85.76 \%$ to $96.02 \%$ and Fig.-6 shows that the highest increase in methyl ester purity was achieved using the 1:5 molar ratio of potassium carbonate to glycerol and the 1:2 mass ratio of ester to DES, which yields a methyl ester with purity of $97.83 \%$, hence it meets the standard to be used as biodiesel.

It can be concluded that DES is able to extract valuable minor components while effectively increase the purity of methyl ester by extracting the impurities from raw methyl ester. 
RASĀYAN J. Chem.

Vol. 11 | No. 4 |1519 - 1524| October - December | 2018

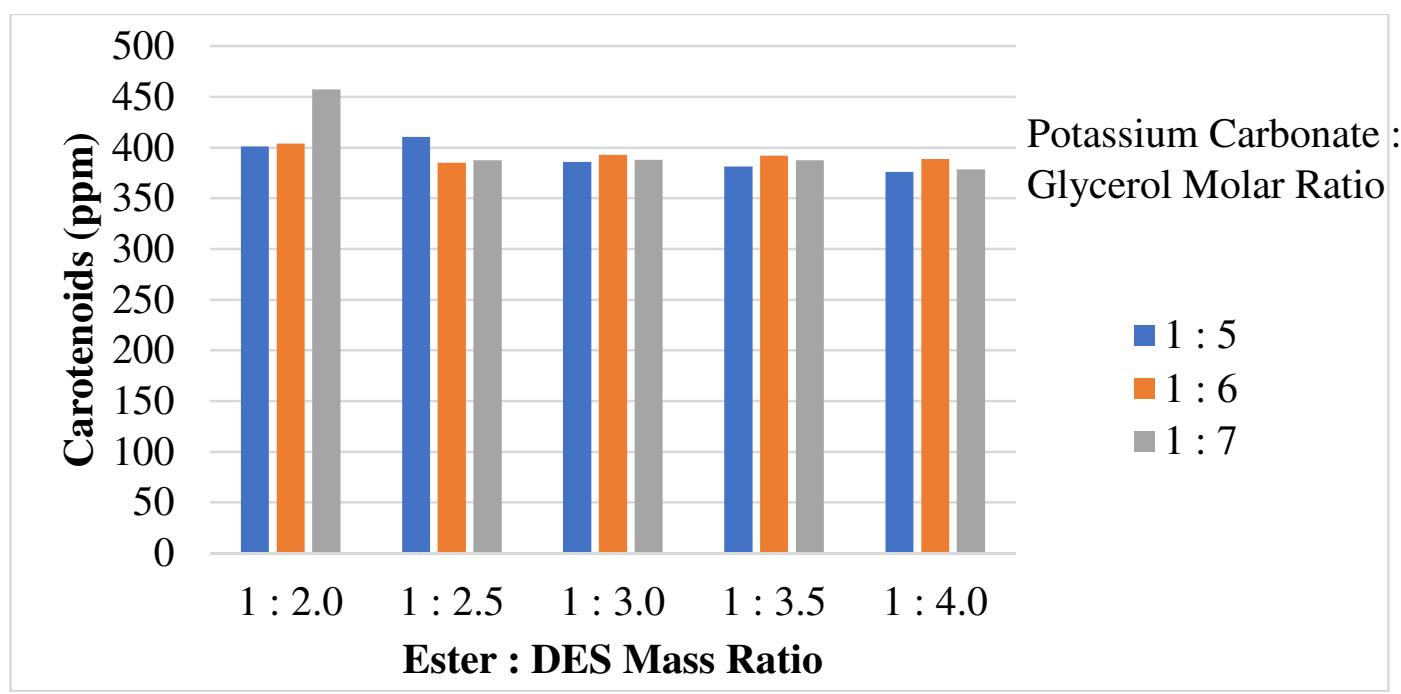

Fig.-4: The Effect of DES on Carotenoids Concentration

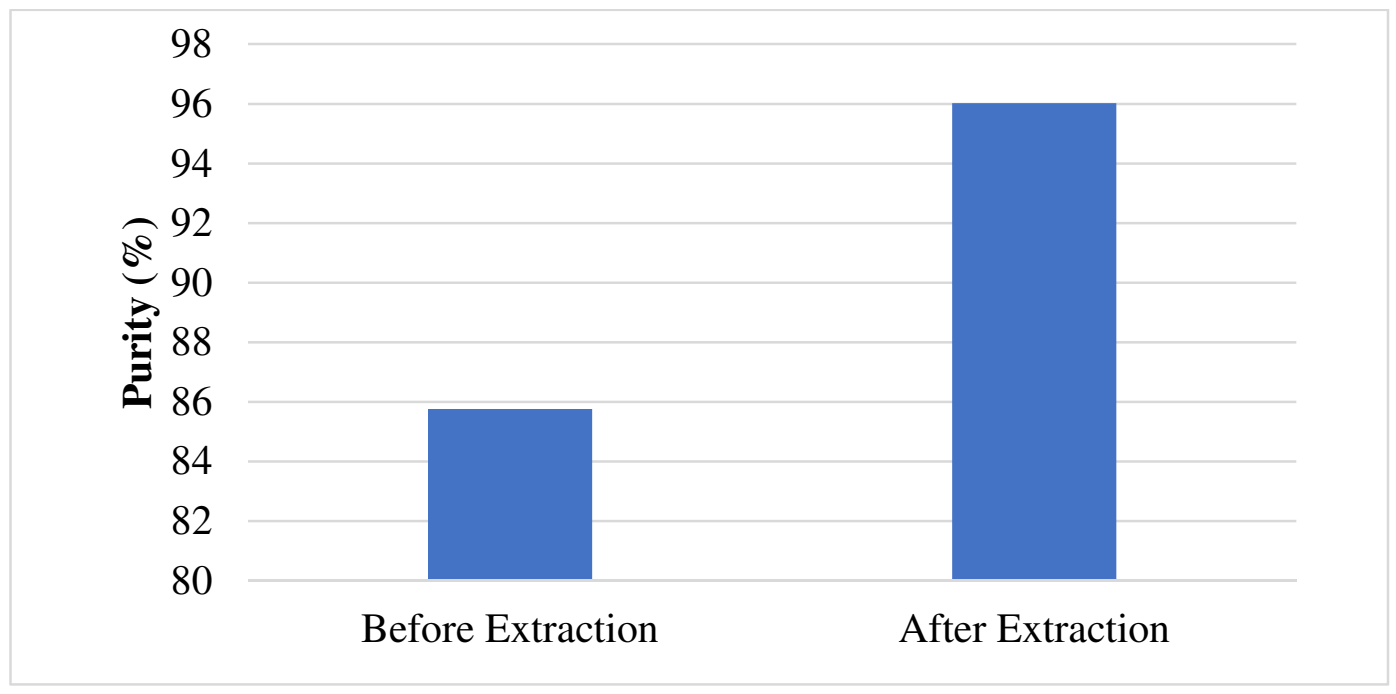

Fig.-5: Overall Purity of Methyl Ester Before and After Extraction

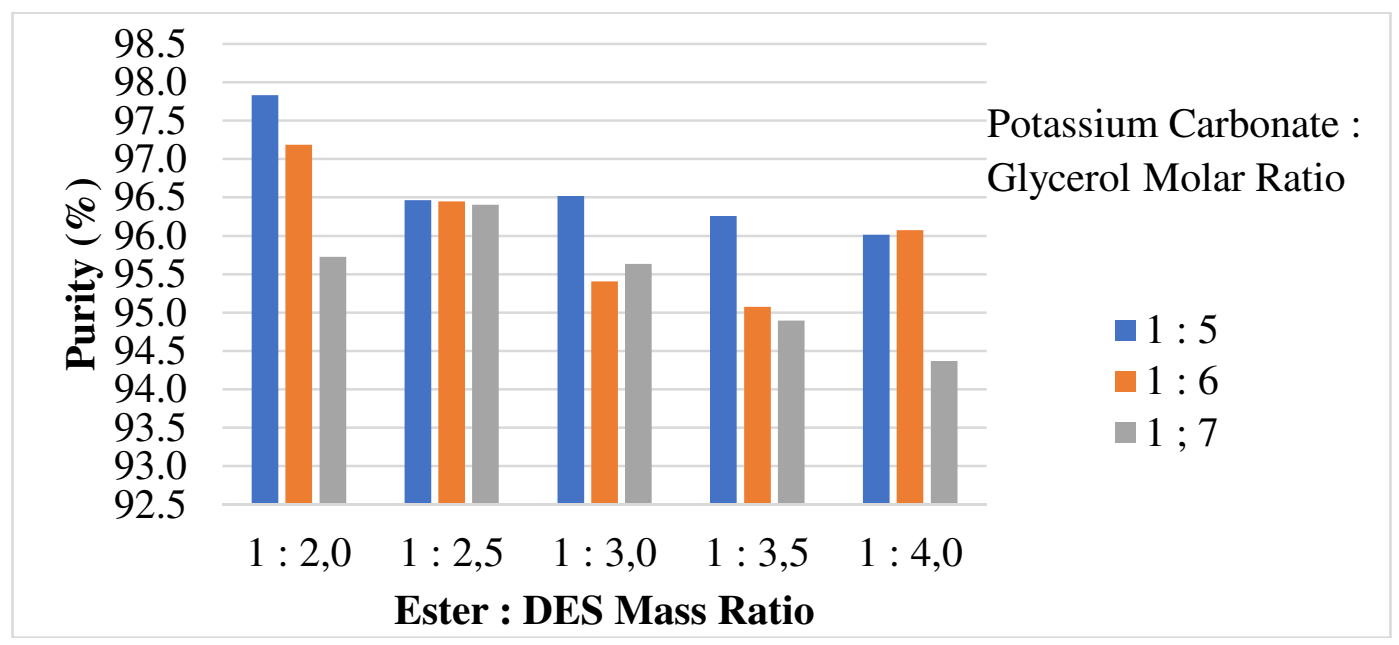

Fig.-6: The Effect of DES on Methyl Ester Purity 


\section{CONCLUSION}

The use of DES to extract minor components indicate that DES is only suitable for extracting components with a hydroxyl group in their molecules, such as vitamin E. However, DES capability of extracting carotenoids from palm methyl ester is far from effective. DES also has the ability to extract impurities from methyl esters, such as waste alcohol, catalyst, glycerol, free fatty acid, monoglycerides and diglycerides, hence increase the purity of methyl ester.

\section{ACKNOWLEDGMENT}

The authors are very grateful to DRPM - the Kemenristek Dikti Republic of Indonesia for funding this research.

\section{REFERENCES}

1 R. Manurung, R. Hasibuan, Taslim, N. Bangun, R. Wulandari and B. I. Sari, ARPN Journal of Engineering and Applied Sciences, 11(16), 9962 (2016).

2 G. Dwivedi, M. P. Sharma and M. Kumar, International Journal of Renewable Energy Research, 4(2), 246 (2014).

3 D. Kumar and B. Verma, Rasayan J. Chem., 11(1), 187 (2018), DOI: 10.7324/RJC.2018.1111813

4 R. S. Raman, G. S. Narayanan, N. Manoharan and S. Sendilvelan, Rasayan J. Chem, 10(3), 944 (2017).

5 W. L. G. da Silva, P. T. de Souza, G.G. Shimamoto and M. Tubino, Braz. Chem. Soc, 26(9), 1745 (2015), DOI:10.5935/0103-5053.20150147.

6 M. H. Hassan and M. A. Kalam, In Proceedings of $5^{\text {th }}$ BSME International Conference on Thermal Engineering, Dhaka, Bangladesh, pp. 39-53 (2013).

7 K. Suppalakpanya, S. Ratanawilai, R. Nikhom and C. Tongurai, Songklanakarin J. Sci. Technol, 33(1), 79 (2011).

8 A. Mancini, E. Imperlini, E. Nigro, C. Montagnese, A. Daniele, S. Orru and P. Buono, Molecules, 20, 17339 (2015), DOI: 10.3390/molecules200917339.

9 NutraSource, Generally Recognized as Safe (GRAS) Determination for the Use of Palm Tocotrienol Rich Fractions (TRF) as Ingredients in Food, MD, 2009.

10 S. R. Valantina, P. A. Sahayaraj and A. A. Prema, Rasayan J. Chem., 3(1), 44 (2010).

11 R. Yusof, E. Abdulmalek, K. Sirat and M. B. A. Rahman, Molecules, 19, 8011 (2014), DOI: 10.3390/molecules19068011.

12 K. Shahbaz, F. S. Mjalli, M. A. Hashim and I. M. AlNashef, Journal of Applied Sciences, 10 (24), 3349 (2010).

13 N. A. Hadi, M. H. Ng, Y. M. Choo, M. A. Hashim and N. S. Jayakumar, J. Am. Oil Chem. Soc., 92(11-12), 1709 (2015), DOI: 10.1007/s11746-015-2720-6.

14 K. C. Ho, K. Shahbaz, W. Rashmi, F. S. Mjalli, M. A. Hashim and I. M. AlNashef, Journal of Engineering Science and Technology, Special issue, 98 (2015).

15 C. W. Puah, Y. M. Choo, A. N. Ma and C. H. Chuah, American Journal of Applied Sciences, 4(6), 374 (2007).

16 K. Sundram, R. Sambanthamurthi and Y. Tan, Asia Pacific J. Clin. Nutr., 12(3), 355 (2003).

17. M. Aamir, M. Ovissipour, S. S. Sablani and B. Rasco, International Journal of Food Science, Article ID 27127(2013), DOI: 10.1155/2013/271271.

[RJC-3079/2018] 